\title{
Weight Management as a Treatment Option for Gastroesophageal Reflux Disease: A Mechanical or Metabolic Rescuer?
}

\author{
Hyuk Lee \\ Department of Medicine, Samsung Medical Center, Sungkyunkwan University School of Medicine, Seoul, Korea
}

\author{
See "Weight Loss as a Nonpharmacologic Strategy for Erosive Esophagitis: A 5-Year Follow-up Study" by Ki Bae Bang, et al. \\ on page 633 , Vol. 12. No. 6,2018
}

Gastroesophageal reflux disease (GERD) is a multifactorial disease with both anatomical and functional factors involved in the pathogenesis. Although the basic understanding is that GERD is caused by increased relaxation of the transient lower esophageal sphincter accompanied by impaired lower esophageal sphincter basal tone or gastric or esophageal motor dysfunction, the exact pathogenesis of GERD is still not fully understood. ${ }^{1}$ In addition, environmental factors are considered as important risk factors for GERD, more specifically, being overweight/obese, inappropriate dietary habits, lack of regular physical activity, and smoking. ${ }^{2}$ Nonetheless, whether or not the improvements or changes in these lifestyle habits can lead to a protective beneficial effect against GERD remains unclear.

American College of Gastroenterology guideline regarding the lifestyle interventions for GERD suggests that weight loss is an effective regimen for GERD symptom improvement and therefore is strongly recommended for patients with body mass index (BMI) $>25 \mathrm{~kg} / \mathrm{m}^{2}$ or with recent weight gain. ${ }^{3}$ Previous randomized controlled trials and prospective population-based cohort studies that assessed the effect of weight reduction for the management of GERD-related symptoms in obese subjects sometimes demonstrated beneficial effects, with normalization of $\mathrm{pH}$ measurements or dose-dependent decrease in symptom prevalence after weight loss. However, other studies suggested that weight loss has no effect on the improvement of GERDrelated symptoms. ${ }^{4}$ Hitherto, the effect of weight loss on GERD is still under debate without conclusion. Although there are multiple reasons for the disagreement, key reasons include the following: the patients also had hiatal hernia, which is the fundamental cause of GERD symptom and therefore cannot be improved with weight loss, and subjective evaluation of the symptoms that vary among different studies. ${ }^{4}$ Overall, studies that focus on a more objective and accurate index-which is endoscopic erosive esophagitis-and its association with weight change are limited.

In this issue of Gut and Liver, Bang and Park ${ }^{5}$ have presented an evidence for the association of BMI with endoscopic erosive esophagitis in their retrospective cohort analysis of the health checkup population. More specifically, improvement of erosive esophagitis was associated with reduced BMI, and adequate weight reduction was positively correlated with more pronounced improvement of erosive esophagitis. Compared to the group with no BMI reduction, the group with BMI reduction exhibited better resolution of erosive esophagitis (odds ratio, 1.44 ; 95\% confidence interval, 1.09 to 1.92 ), indicating the clinical significance of these findings. Furthermore, these effects are more pronounced in obese subjects. The main strengths of this study are as follows: a longitudinal analysis of exceptionally large population for a 5-year follow-up duration; the effect of weight management evaluated by objective improvement of erosive esophagitis; and the study cohort being a health checkup population, allowing for adjustment of various confounding parameters. Most interestingly, in the subgroup with BMI reduction $\geq 1$, persistently significant resolution effect of erosive esophagitis was observed even after adjusting for various metabolic parameters (i.e., lipid profile or homeostatic model assessment for insulin resistance). This suggests that correction of obesity without metabolic regulation is sufficient for adequate

Correspondence to: Hyuk Lee

Division of Gastroenterology, Department of Medicine, Samsung Medical Center, Sungkyunkwan University School of Medicine, 81 Irwon-ro, Gangnam-gu, Seoul 06351, Korea

Tel: +82-2-3410-3409, Fax: +82-2-3410-6983, E-mail: leehyuk@skku.edu

pISSN 1976-2283 eISSN 2005-1212 https://doi.org/10.5009/gnl18451

@) This is an Open Access article distributed under the terms of the Creative Commons Attribution Non-Commercial License (http://creativecommons.org/licenses/by-nc/4.0) which permits unrestricted non-commercial use, distribution, and reproduction in any medium, provided the original work is properly cited. 
beneficial effect. However, assessing long-term effects beyond the current follow-up duration can lead to different outcomes, and improvement of long-term obesity will maximize the regulation of metabolic syndrome, resulting in a greater improvement of erosive esophagitis.

In fact, the pathophysiological mechanism of erosive esophagitis in obese or overweight population may involve both the mechanical cause-relaxation of lower esophageal sphincter induced by an increased abdominal pressure-and other coexisting metabolic syndrome as another cause. Visceral fat secretes inflammatory mediators, cytokines, and insulin-like growth factors to induce a systemic inflammatory and insulin-resistant state, which may be associated with esophagitis, although there is insufficient evidence. ${ }^{6,7}$ Nonetheless, a previous study assessing the association between BMI and the occurrence of erosive esophagitis demonstrated that cardiometabolic risk factors greatly contributes (>35\%) to the occurrence of GERD, partially or fully mediating the association between overweight/obesity and incident erosive esophagitis. ${ }^{8}$ The conclusion by Bang et al. needs to be further specified with consideration of these findings. In other words, in order to identify whether the improvement of erosive esophagitis via weight reduction is solely due to the weight reduction or is through metabolic regulation, analysis of subgroups (metabolic unhealthy obesity and metabolic healthy obesity) is required. Based on the findings of this subgroup analysis, management of erosive esophagitis in each subgroup can be differentially performed by therapeutically targeting the BMI or directly targeting the metabolic syndrome.

In conclusion, aside from pharmacologic or endoscopic treatment, the importance of weight management should be primarily considered as the foundation of the treatment of erosive esophagitis. More specifically, life style modification in obese subjects is more important than other treatments. Indeed, the pathophysiologic aspect of treatment effectiveness still remains unclear, and improvement from BMI reduction to the mediating effect of metabolic syndrome should be simultaneously considered and performed in future studies.

\section{CONFLICTS OF INTEREST}

No potential conflict of interest relevant to this article was reported.

\section{REFERENCES}

1. Boeckxstaens G, El-Serag HB, Smout AJ, Kahrilas PJ. Symptomatic reflux disease: the present, the past and the future. Gut 2014;63:1185-1193.

2. Festi D, Scaioli E, Baldi F, et al. Body weight, lifestyle, dietary habits and gastroesophageal reflux disease. World J Gastroenterol 2009;15:1690-1701.

3. Katz PO, Gerson LB, Vela MF. Guidelines for the diagnosis and management of gastroesophageal reflux disease. Am J Gastroenterol 2013;108:308-328.

4. Ness-Jensen E, Hveem K, El-Serag H, Lagergren J. Lifestyle intervention in gastroesophageal reflux disease. Clin Gastroenterol Hepatol 2016;14:175-182.e3.

5. Bang KB, Park JH. Weight loss as a nonpharmacologic strategy for erosive esophagitis: a 5-year follow-up study. Gut Liver 2018;12:633-640.

6. Fass R. The pathophysiological mechanisms of GERD in the obese patient. Dig Dis Sci 2008;53:2300-2306.

7. Souza RF, Huo X, Mittal V, et al. Gastroesophageal reflux might cause esophagitis through a cytokine-mediated mechanism rather than caustic acid injury. Gastroenterology 2009;137:1776-1784.

8. Lee H, Lim Y, Chi S, et al. Relationship between obesity and development of erosive reflux disease: a mediation analysis of the role of cardiometabolic risk factors. Sci Rep 2017;7:6375. 